\title{
Fluid flow through nanometer-scale channels
}

\author{
J.-T. Cheng and N. Giordano \\ Department of Physics, Purdue University, West Lafayette, Indiana 47907-1396
}

(Received 20 July 2001; published 7 March 2002)

\begin{abstract}
We describe studies of the pressure driven flow of several classical fluids through lithographically produced channels in which one dimension, the channel height $h$, is in the micron or nanometer size range. The measured flow rates are compared with theoretical predictions assuming no-slip boundary conditions at the walls of the channel. The results for water agree well with this prediction for $h$ as small as $40 \mathrm{~nm}$ (our smallest channels). However, for hexane, decane, hexadecane, and silicone oil we find deviations from this theory when $h$ is reduced below about $100 \mathrm{~nm}$. The observed flow rates for small $h$ are larger than theoretical expectations, implying significant slip at the walls, and values of the slip length are estimated. The results are compared with previous experimental and theoretical work.
\end{abstract}

DOI: 10.1103/PhysRevE.65.031206

PACS number(s): 47.15.Rq

\section{INTRODUCTION AND BACKGROUND}

The pressure driven flow of a classical Newtonian fluid, such as water, in a macroscopic container can usually be described by Poiseuille's law. This law follows routinely from the Navier-Stokes equations together with some assumptions about the nature of fluid motion at the walls of the container $[1,2]$. It is customary to assume that the tangential fluid velocity vanishes at a wall, i.e., no-slip boundary conditions. While this is not likely to be correct at very small (i.e., molecular) length scales (e.g., [3-6]), it is believed to be accurate at "macroscopic" scales. It was, therefore, quite surprising when recent experimental studies of silicone oil and isopropol alcohol reported a breakdown of Poiseuille's law in channels in which the smallest dimension $h$ was of order of $20 \mu \mathrm{m}$ [7-11]. Interestingly, some (but not all) of these experiments observed smaller flow rates than expected with no-slip boundary conditions, so simply incorporating slip into the theory cannot account for those experiments.

The present paper was designed to investigate this apparent breakdown of Poiseuille's law in more detail, by extending the experiments to much smaller length scales. Our major results are as follows. (1) We see no evidence for a breakdown of Poiseuille's law in "large" channels, i.e., in channels with dimensions comparable to those studied in Refs. [7-11]. (2) We do observe deviations from Poiseuille's law in much smaller channels, and these deviations depend systematically on the size of the fluid molecule. (3) The deviations we observe correspond to flow rates that are larger than predicted by the theory with no-slip boundary conditions. Hence, our results indicate that slip is significant, and allow us to estimate the slip length.

\section{SAMPLE FABRICATION}

Figure 1 shows how the samples were constructed. The bottom surface and walls of the desired flow channel pattern were produced by exposure and development of a layer of photoresist. The top of the flow channel was formed by sealing with a second substrate that had been freshly coated with second layer of photoresist, which acted essentially as a "glue" to bond the top plate to the body of the structure.
Fluid was injected into and removed from the channel through holes drilled into the top plate prior to this bonding. The following paragraphs describe the fabrication process in more detail.

The bottom substrate in Fig. 1 was a 0.25 -mm-thick cover glass, which was first coated with a layer of photoresist. The thickness of this photoresist would eventually become the height of the flow channel, $h$. This height was varied by using different types of photoresist, employing different spin rates for depositing the photoresist, and by diluting the photoresist with a thinning solution [12]. In this way $h$ was varied from $2.7 \mu \mathrm{m}$ to $40 \mathrm{~nm}$. This photoresist layer was exposed and developed in two stages. It was first exposed through a contact mask and then developed so as to open up two large regions, which would later become inlet and outlet regions leading to and starting from the flow channel. These inlet and outlet regions were positioned to overlap with inlet and outlet holes drilled in the top plate. The inlet and outlet regions also contained cylindrical "pillars" to prevent the top and bottom plates from touching in these regions under the pressure applied during bonding.

The flow channel was formed by a second exposuredevelopment step. For this case the desired flow channel pattern (typically one or a few straight channels) was projected onto the substrate using an optical microscope [13]. The channel width $w$ was varied from a few to $20 \mu \mathrm{m}$, with the larger values of $w$ usually corresponding to the thinnest channels (smallest $h$ ), so as to keep the flow rate in a convenient range. However, in many cases we also varied $w$ with $h$ kept approximately constant to check that the variations we observed were correlated with $h$ and not $w$. The channel length was in the range 100-900 $\mu \mathrm{m}$. This was sufficiently long that the pressure variation within the inlet and outlet regions combined was never more that $5 \%$ of the pressure across the channel. These inlet and outlet pressure drops were estimated theoretically [14], and the final pressure data corrected to correspond to just the pressure across the flow channel.

The top surface of the flow structure was sealed with a glass coverplate $(0.25 \mathrm{~mm}$ thick), which had been coated with a thin (200-nm) layer of (unbaked) photoresist just prior to bonding to the main substrate. This bonding was accom- 


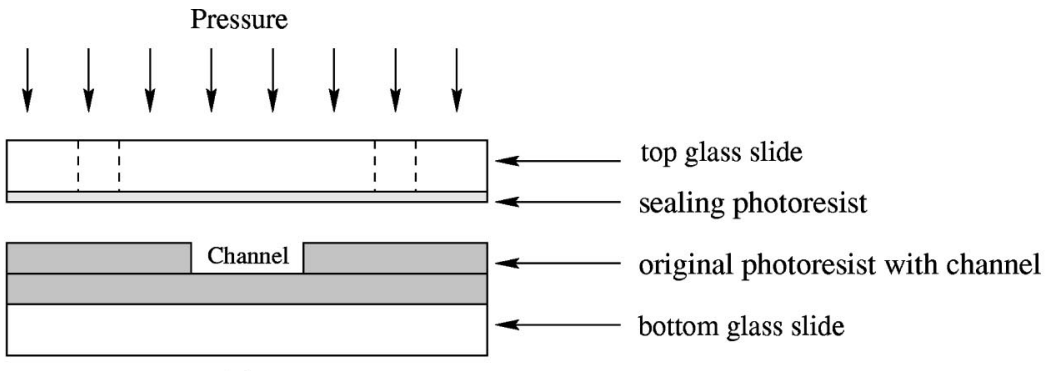

(a)

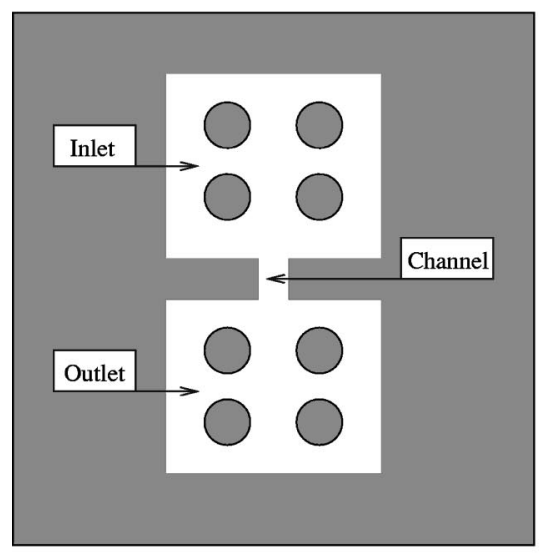

(b) bottom plate

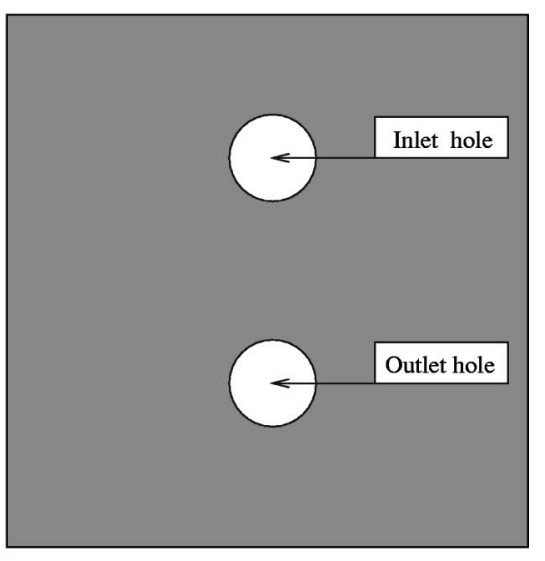

(c) top plate
FIG. 1. Schematic of the sample fabrication procedure, not to scale. (a) Side view of the top and bottom plates just prior to bonding. (b) Top view of the bottom plate pattern. The pillars in the inlet and outlet regions are shown. (c) Top view of the top plate pattern. plished with gentle pressure from a vacuum holder. The quality of the seal could be judged easily by inspection with an optical microscope-leaks (if present) were clearly visible through variations in the optical opacity, and such samples were discarded.
An accurate measure of the channel height was essential for calculating the expected Poiseuille flow rate. This height was determined in two independent ways. First, the thickness of the bottom photoresist layer was determined from the manufacturer's specifications, using the known photoresist

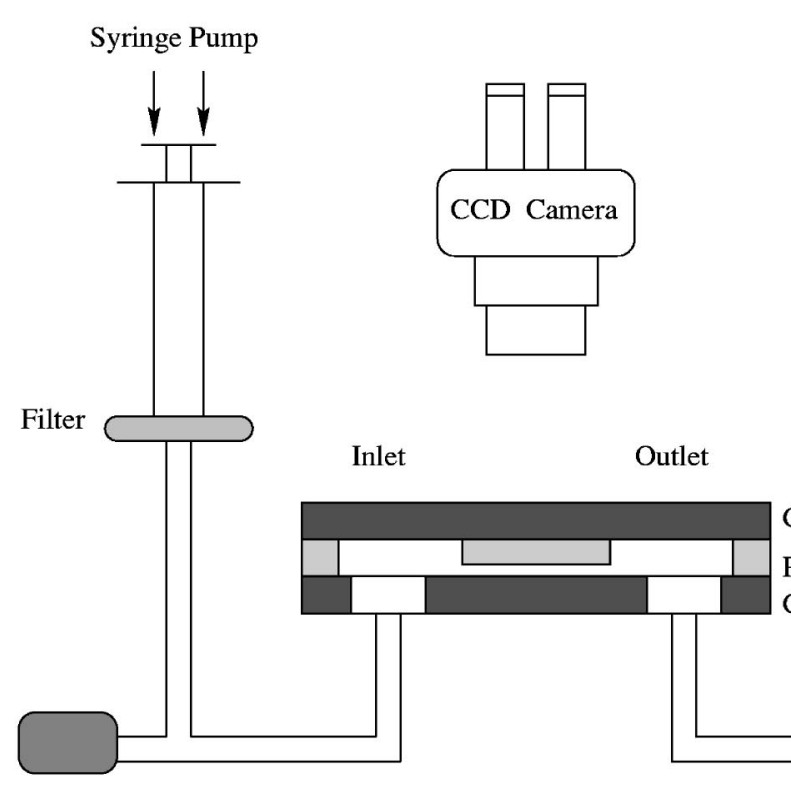

Pressure Gauge

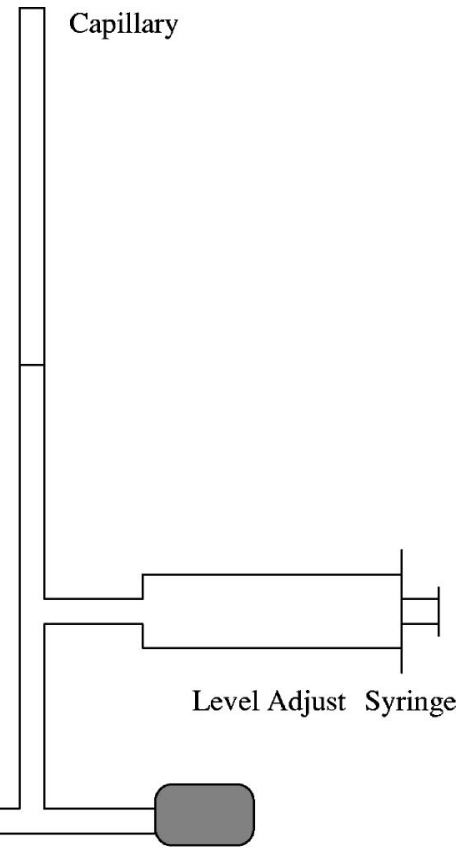

Pressure Gauge

FIG. 2. Schematic the system used to measure the flow rate. The flow rate is measured by observing the motion of the meniscus in an outlet capillary, which is here a macroscopic capillary. For some samples a second flow channel, fabricated on the substrate along with the sample, was used. 
spin rate and the concentration of the thinning solution (if any was used). Second, in some samples we deposited thin metal film electrodes in the inlet and outlet regions. The channel was then filled with an electrolyte (dilute nitric acid), and the measured conductance used to infer the channel height (the width and length were measured using microscopy). The channel height measured in this way agreed with the value expected from the manufacturer's estimate of the photoresist thickness to within the combined uncertainties of approximately $\pm 5 \%$. Since the (theoretical) Poiseuille rate varies as $h^{3}$, this contributes a $15 \%$ uncertainty in the theoretically expected flow rate, and will turn out to be a major source of error.

Atomic force microscopy measurements were used to characterize the roughness of the top and bottom surfaces of the flow channel. In both cases the peak-to-peak roughness was $\approx 0.5 \mathrm{~nm}$ over distances of order of $1 \mu \mathrm{m}$. This roughness may be important in theoretical estimates of the slip length.

\section{APPARATUS}

The apparatus shown in Fig. 2 was used to measure the flow rate, as fluids moved through the sample in response to an imposed pressure. This pressure was applied using a simple syringe, and measured with one or more piezoelectric pressure gauges. Typical pressures were of order of $1 \mathrm{~atm}$. The inlet line contained a $25-\mathrm{nm}$ filter; with this filter in place, blockage was not a problem for the time required to study a sample, typically $1 \mathrm{~h}$.

For the larger channels the flow rate could be measured by simply monitoring the liquid level as it moved through a macroscopic capillary (0.4-mm diameter), which was in series with the outlet side of the sample. With the smallest channels the flow rate was too small to measure conveniently in this way. Instead, we used a second channel that had been fabricated in series, and on the same substrate, with the sample. The motion of fluid through this measurement channel was measured with photomicroscopy.

Since the photoresist used to bond the cover plate to the substrate was never "baked" (in the sense of standard photoresist processing), care was taken so that the samples were only exposed to red light. This applied to storage prior to or after measurement, and to the photomicroscopy mentioned in the previous paragraph. Exposure to white or blue light caused the formation of pockets of $\mathrm{N}_{2}$ gas in the unbaked photoresist layer, as part of the normal photochemical reactions, which occur during photoresist processing [15]. The size of these pockets depended on the exposure conditions (time and temperature), and (if they were allowed to form) were typically many microns in diameter.

All of the measurements were conducted at room temperature $\left(25^{\circ} \mathrm{C}\right)$, and the theoretically expected Poiseuille flow rates were calculated using values of the viscosity measured at this temperature.

\section{RESULTS}

The measurements were carried out by first filling the sample with fluid with a constant pressure applied at the

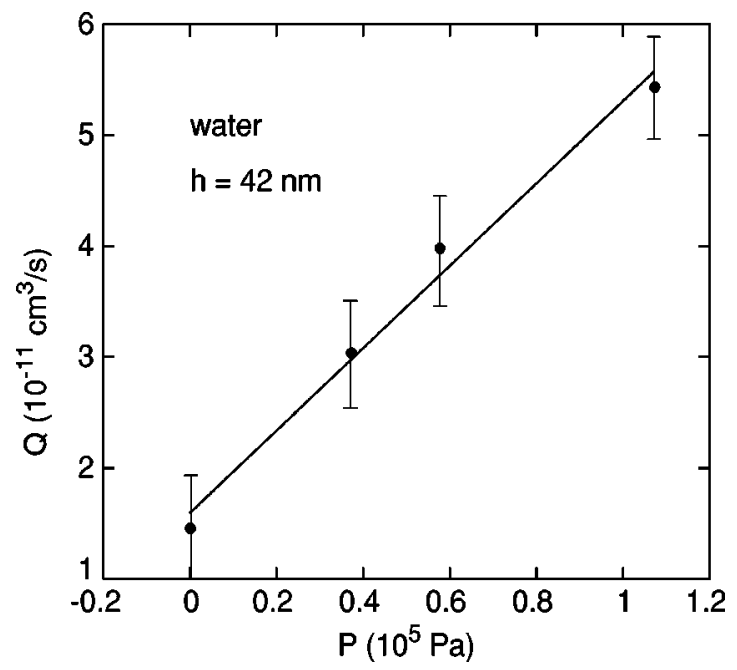

FIG. 3. Flow rate $Q$ as a function of pressure for water for a sample with a channel of height $h=42 \mathrm{~nm}$. The channel width and length were $7.5 \mu \mathrm{m}$ and $118 \mu \mathrm{m}$, respectively. The solid line is a least squares fit.

inlet. The outlet pressure was always atmospheric pressure. Values of the sample pressure $P$ given below are always the difference between the inlet and outlet pressures, corrected for the estimated pressure drop in the inlet and outlet regions (as mentioned above). The position $z$ of the fluid meniscus in the outlet capillary (either the external capillary or the measurement capillary fabricated on the substrate) was measured as a function of time. $z$ was always found to be a linear function of time (as expected), confirming that sample blockage was not a problem.

The slope of the $z-t$ relation was obtained from a least squares fit, yielding the volume flow rate $Q$ at a particular value of $P$. Measurements at different values of $P$ were then performed, and it was found that, again as expected, $Q$ varied linearly with $P$ to within experimental error. An example of this behavior is shown in Fig. 3. Note that $Q$ is nonzero at $P=0$, due to the capillary pressure in the measurement capillary. These data were obtained using a measurement capillary on the substrate, which, as noted above, was in series with the flow channel and located between this channel and the outlet region. This capillary had the same height as the sample (in this case $h=42 \mathrm{~nm}$ ), so the associated capillary pressure acted to draw fluid into the flow channel, hence the negative intercept with the pressure axis in Fig. 3. An estimate of this capillary pressure is made difficult by the fact that one of the surfaces is photoresist (the other is glass) and the associated surface tension is not known (we hope to measure this quantity in the near future). Meanwhile, if we assume that the value of this surface tension is the same as for glass, we can estimate the capillary pressure using the usual relation $P_{\text {cap }} \sim 2 \gamma / h$, where $\gamma$ is the surface tension. For this sample we find the theoretical value $P_{c a p}=2.3 \times 10^{4} \mathrm{~Pa}$, in good agreement with the magnitude of $P$ at which $Q$ extrapolates to zero in Fig. 3.

According to the theory of Poiseuille flow, with no-slip boundary conditions at the channel walls, the volume flow rate for a channel whose width and length are much greater 


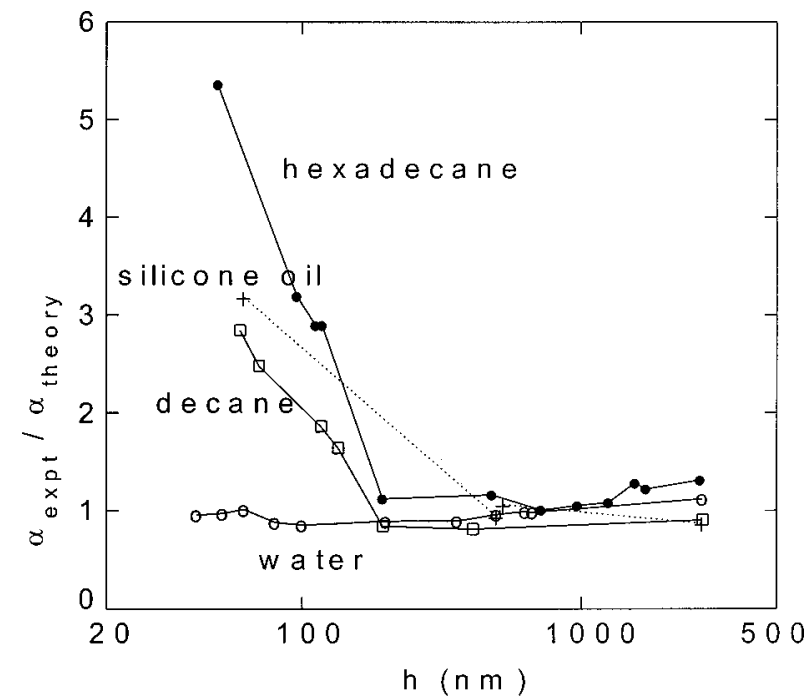

FIG. 4. $\alpha_{\text {expt }} / \alpha_{\text {theory }}$, the experimental flow rate normalized by the theoretical prediction for Poiseuille flow with no-slip boundary conditions, as a function of channel height $h$ for several fluids. Typical error bars are approximately the size of the symbols, the lines are guides to the eye.

than the height is given by [1]

$$
Q_{\text {theory }}=\frac{h^{3} w}{12 \mu L} P,
$$

where $\mu$ is the viscosity, and $P$ is again the pressure across the channel; note that Eq. (1) assumes that there is no contribution from capillary pressure as mentioned above. Hence, the theory predicts that for our case $Q_{\text {theory }}=\alpha_{\text {theory }}(P$ $\left.-P_{0}\right)$ with

$$
\alpha_{\text {theory }}=\frac{h^{3} w}{12 \mu L}
$$

and with a nonzero $P_{0}$ due to capillary pressure effects. The slope of the $Q-P$ relation, as in Fig. 3 , thus yields the experimental value of $\alpha$.

Figure 4 shows results for several fluids, where we plot the ratio of the experimentally measured and theoretically predicted values of $\alpha$ as a function of channel height $h$ [16]. Note that each data point was obtained from the average flow rate of typically 15 separate samples, which had different values of $w$ (but the same value of $h$ ). The main contributions to the overall are from the theoretical estimate of $\alpha$ (through the uncertainty in the measured $h$ ) and the uncertainty in the measurement of $Q$.

For water the experimental and theoretical values of $\alpha$ are equal (to within the uncertainties) at all values of $h$. Hence, there is no evidence for slip in this case. However, the picture is different for the other fluids. The fluid with the largest molecular size is hexadecane, and it exhibits the largest deviation from the no-slip theory. Decane is a smaller molecule in the same family and it shows smaller deviations from the no-slip theory. We also have limited results for hexane (not shown here), an even smaller molecule from this series, which indicate even smaller deviations from the theory. This trend suggests that the slip length varies systematically with the molecular size, which seems quite reasonable. The last fluid considered in Fig. 4 is silicone oil. We have studied this fluid so as to be able to compare directly with the earlier experiments discussed in the introduction. We find deviations from the no-slip theory only for $h$ below about $300 \mathrm{~nm}$. This contrasts sharply with the previous results [7-11], which suggested deviations for much (more than an order of magnitude) larger values of $h$. The reason for this discrepancy is not clear to us.

\section{DISCUSSION}

The results in Fig. 4 show that the flow rates for decane, silicone oil, and hexadecane deviate increasingly from the no-slip theory as $h$ is reduced. One can go beyond the no-slip theory in several ways. It is perhaps simplest to assume a nonzero (and phenomenological) slip length $\lambda[3-6]$. This length can be defined through the velocity profile across the channel. For Poiseuille flow through a narrow channel one finds [1]

$$
u(z)=\beta\left(h^{2}-4 z^{2}\right),
$$

where $u$ is the velocity, $\beta$ is a numerical factor involving the viscosity and the pressure gradient, and the channel runs between $\pm h / 2$ in the $z$ direction (we also assume that $h$ is much less than the length and width of the channel). The flow rate $Q$ is then obtained by integration of Eq. (3) over the range of the channel $-h / 2<z<h / 2$.

If there is slip at the walls of the channel, it is natural to define the slip length through (see also [17])

$$
u(z)=\beta\left[(h+2 \lambda)^{2}-4 z^{2}\right] .
$$

Now $u$ vanishes not at the walls, but at a fictitious point a distance $\lambda$ outside the channel. Integrating Eq. (4) we find the flow rate

$$
Q_{\text {slip }}=Q_{0}\left(1+\frac{6 \lambda}{h}\right)
$$

where $Q_{0}$ is the flow rate in the absence of slip, and we have assumed $\lambda / h \ll 1$. (Note that it is not really necessary to make this assumption; we have in fact used the full expression for the flow rate, the integral of Eq. (4) in analyzing our data below.) Since the channel height, the fluid viscosity, and other relevant parameters are all known, the experimental results in Fig. 4 can be used with Eq. (5) (or its more general form) to obtain values of the slip length as a function of $h$. If this description of the flow behavior makes sense, we should find that $\lambda$ is independent of $h$.

Results for the slip length as a function of $h$ are shown in Fig. 5. Only for decane and hexadecane do we have sufficient data to examine the variation with $h$ in any detail. For decane $\lambda$ does indeed appear to have a constant value, which is near $14 \mathrm{~nm}$ over a wide range of $h$. For hexadecane it appears that $\lambda$ decreases somewhat as $h$ is reduced. This may indicate that the fluid behavior near the wall is too compli- 


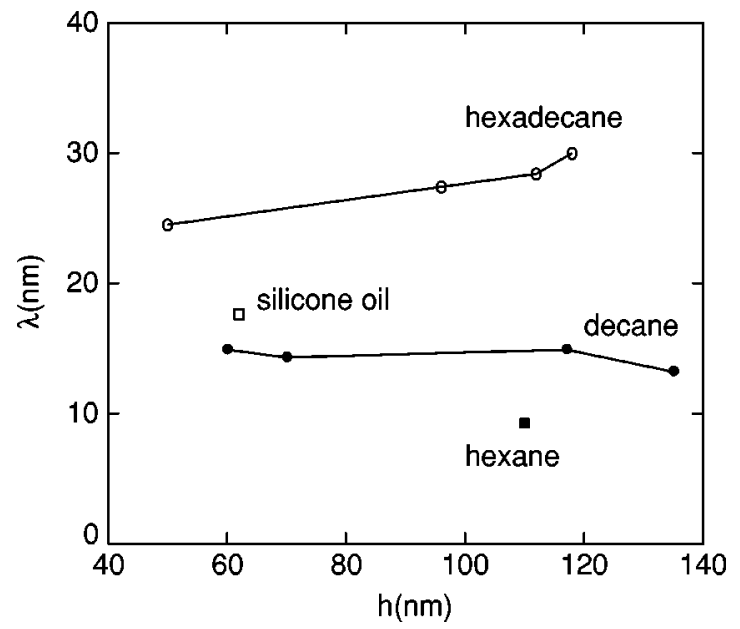

FIG. 5. Slip length $\lambda$ as a function of channel height $h$ for several fluids. The lines are guides to the eye.

cated to be described by a slip length with a velocity profile as simple as Eq. (4), but this apparent trend could also be due to uncertainties in $\lambda$.

While the question of slip at a liquid-solid interface has been much discussed, there is little quantitative experimental evidence for a nonzero slip length in nonpolymeric fluids. Recently, Pit and co-workers [17] have used a near-field optical technique [18] to measure the slip length of hexadecane when flowing over sapphire surfaces that were pretreated in different ways. For a clean surface they found $\lambda=(175$ $\pm 50) \mathrm{nm}$, while surfaces coated in various ways yielded values ranging from $0-400 \mathrm{~nm}$. These values are much greater than the molecular size, which is of order a few nanometers, but there does not appear to be a fundamental reason why $\lambda$ must be closely related to this number. Our results for hexadecane (Fig. 5) are in the range $\lambda$ $\approx 25-30 \mathrm{~nm}$, which is much smaller than found by Pit and co-workers for a clean surface. However, our paper did not involve sapphire surfaces, so a more direct comparison is not possible at this time.

On the theoretical side, there have been molecular dynamics studies of slip behavior in Lennard-Jones fluids [5], and also in hexadecane [6]. Both calculations observe slip and find, not surprisingly, that the slip length depends on the strength of the fluid-wall interaction. Unfortunately, the theory is not yet able to estimate this interaction strength for realistic walls (i.e., glass or sapphire), so a direct comparison with the experiments is not possible at present.

\section{CONCLUSIONS}

We have devised a method for making liquid flow channels in which one dimension $h$ is in the nanometer range. These channels have been used to study the pressure driven flow of several Newtonian fluids, and we have obtained strong evidence for slip at the interface between the fluid and the walls of the flow channel. We find a slip length $\lambda$ $\approx 25-30 \mathrm{~nm}$ for hexadecane, $\sim 14 \mathrm{~nm}$ for decane, and $\sim 9 \mathrm{~nm}$ for hexane. It will be interesting to extend these measurements to other fluids, and to also study the effect of surface preparation on $\lambda$.

\section{ACKNOWLEDGMENTS}

We thank G. Fiete and M. Dorbin for their initial involvement in the experiment. This work was supported by NSF Grant No. DMR-9970708 and by DOE Contract No. DEAC26-99BC15207.
[1] F.M. White, Viscous Fluid Flow (McGraw-Hill New York, 1974).

[2] D. J. Tritton, Physical Fluid Dynamics (Clarendon Press, Oxford, 1988).

[3] J. Koplik, J.R. Banavar, and J.F. Willemsen, Phys. Fluids A 1, 781 (1989).

[4] J. Koplik and J.R. Banavar, Annu. Rev. Fluid Mech. 27, 257 (1995).

[5] P.A. Thompson and S.M. Troian, Nature (London) 389, 360 (1997).

[6] M.J. Stevens, M. Mondello, G.S. Grest, S.T. Cui, H.D. Cochran, and P.T. Cummings, J. Chem. Phys. 106, 7303 (1997).

[7] J. Harley, H. Bau, J.N. Zemel, and V. Dominko Proc-IEEE Micro Electro Mech.Sys. 89THO249-3, 25 (1989).

[8] J. Pfahler, J.C. Harley, and H. Bau, Sens. Actuators A 21, 431 (1990).

[9] J. Pfahler, J. Harley, H. Bau, and J.N. Zemel, Micromech. Sens. Actuators Sys. 32, 49 (1991).

[10] J. N. Pfahler, Ph.D. thesis, University of Pennsylvania, 1992.

[11] W. Urbanek, J.N. Zemel, and H.H. Bau, J. Micromech. Mi- croeng. 3, 206 (1993).

[12] Microelectronic Product Guide (Shipley Co., Newton, MA, 1982).

[13] D.W. Palmer and S.K. Decker, Rev. Sci. Instrum. 44, 1621 (1973).

[14] S. E. Koonin and D. C. Meredith, Computational Physics (Addison-Wesley, Reading, 1990).

[15] Introduction to Microlithography, 2nd ed., edited by L. F. Thompson, C. G. Wilson, and M. J. Bowden (American Chemical Society, Washington, DC, 1994).

[16] All of the measurements were conducted at room temperature, and the handbook values of the viscosity were used in evaluating the theory. The values we used are $0.896 \mathrm{cp}$ for water, $0.312 \mathrm{cp}$ for hexane, $0.885 \mathrm{cp}$ for decane, $3.18 \mathrm{cp}$ for hexadecane, and $96.4 \mathrm{cp}$ for silicone oil. We also measured the viscosities in our lab, and found values consistent with these.

[17] R. Pit, H. Hervet, and L. Léger, Phys. Rev. Lett. 85, 980 (2000).

[18] R. Pit, H. Hervet, and L. Léger, Tribol. Lett. 7, 147 (1999). 